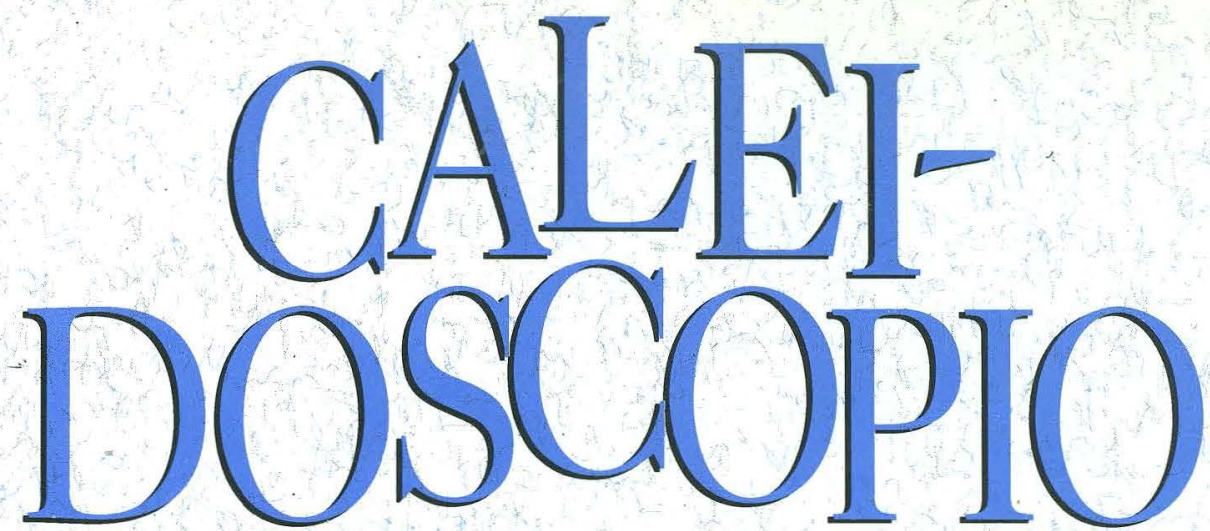

REVISTA SEMESTRAL DE CIENCIAS SOCIALES Y HUMANIDADES

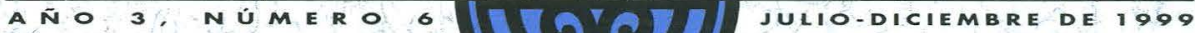

Descentralización e innovación educativa en Aguascalientes: 1992-1998

MARGARITA ZORIILLA FIERRO

Tocqueville se detwo en Texas

CESAR GILABETT

El problema de la representación: notas para el análisis de la religiosidad popular de los mexicanos en el exilio a luis rodolfo moran euroz

La Virgen de Guadalupe en Zacatecas: una imagen en pugna

MARLANA TERAN PUENTES

El territorio de Jalisco - José maría múria

Los derechos humanos, desde el iusnaturalismo y el personalismo

JESUS ANTONIO DE LA TORIE RANGEL

Kierkegaard: la angustia y lo trágico de la modernidad

GRESCENCIANO GRAVE TIRADO:

Algunas consideraciones sobre la ética nietzscheana

IENRIQUE LUJAN SAINAAR

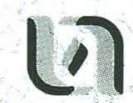

UnIUERSIDad aUTONOMa DE aGUaSCaLIERTES 


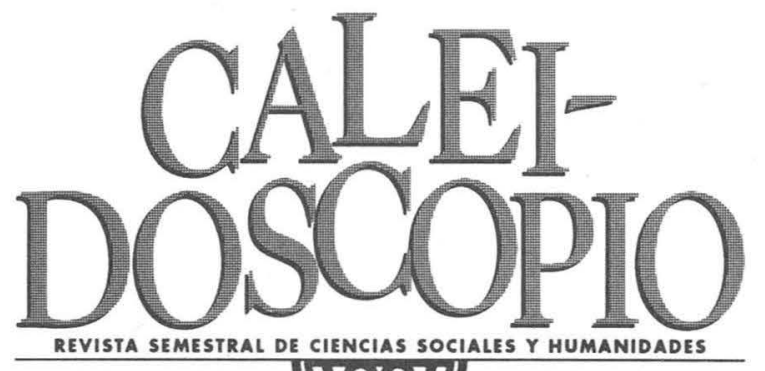

世. 



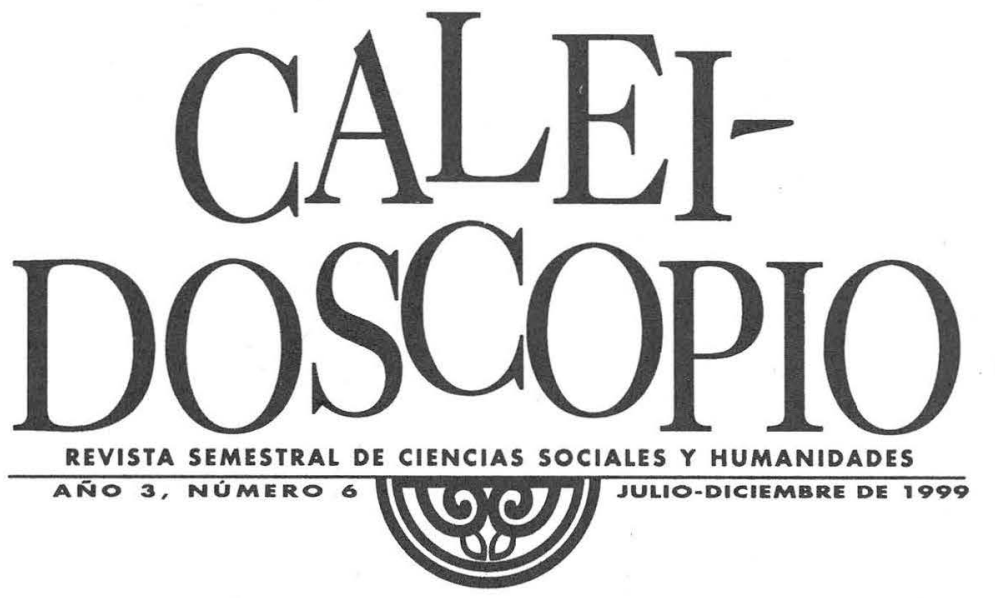


educativas en Aguascalientes:

1992-1998. Una mirada desde dentro

$\begin{array}{rr}\text { CÉSAR } & 65 \\ \text { GILABERT } & \end{array}$

ocqueville se detuvo en Texas

MARIANA

TERÁN

FUENTES

una imagen en pugna

Notas para el análisis de la religiosidad

RODOLFO

MORÁN

QUIROZ

popular de los mexicanos en el exilio

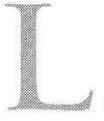

a Virgen de Guadalupe en Zacatecas:

l problema de la representación.

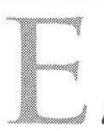

I territorio de Jalisco

JOSÉ $\mathbf{1 3 5}$
MARÍA
MURIÁ

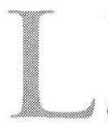

os derechos humanos, desde

el iusnaturalismo y el personalismo

$$
\begin{array}{r}
\text { JESÚS } \\
\text { ANTONIO } \\
\text { DE LA TORRE } \\
\text { RANGEL }
\end{array}
$$$$
147
$$
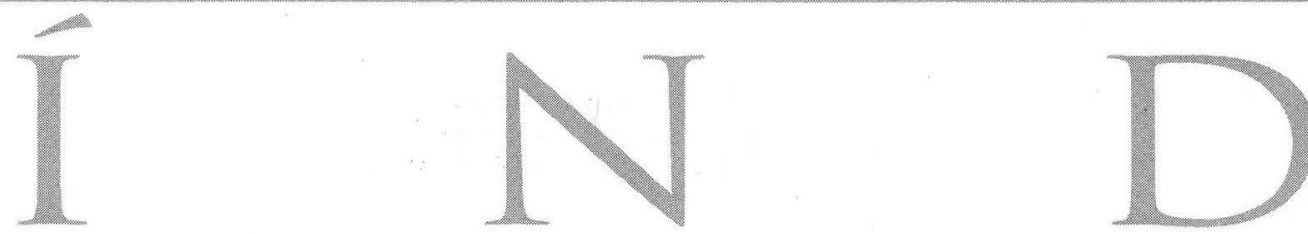


\section{y lo trágico en la modernidad}

\section{ENRIQUE}

LUJÁN

193

SALAZAR

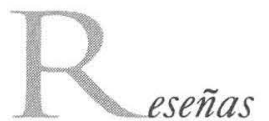

\section{ared Diamond}

ARMAS, GEERMENES Y ACERO, LA SOCIEDAD HUMANA Y SUS DESTINOS, MADRID, EDITORIAL DEBATE, 1998, 527 PÁGS. (PRIMERA EDICIÓN EN INGLÉS: 1997).

FELIPE MARTÍNEZ RIZO

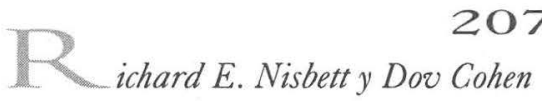

CULTURE OF HONOR. THE PSYCHOLOGY OF VIOLENCE IN THE SOUTH, BOULDER, CO. WESTVIEW PRESS, 1996, XVIII + 119 PÁGS.

FELIPE MARTINEZ RIZO

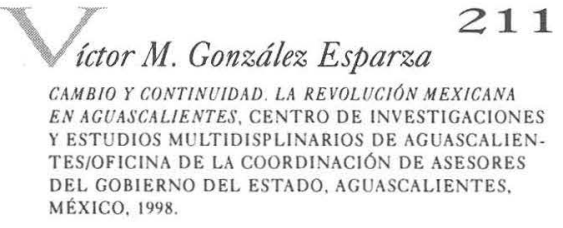

YOLANDA PADILLA RANGEL

\section{Seatriz Rojas}

LAS INSTITUCIONES DE GOBIERNO Y LA ÉLITE LOCAL. AGUASCALIENTES DEL SIGLO XVII HASTA

LA INDEPENDENCIA, MÉXICO, EL COLEGIO

DE MICHOACÁN-INSTITUTO MORA, 1998, 339 PP.

\section{JESÚS GÓMEZ SERRANO}

\section{osé Antonio Gutiérrez $G$.}

\section{1}

HISTORIA DE LA IGLESIA CATÓLICA EN AGUASCALIENTES. VOLUMEN I: PARROQUIA DE LA ASUNCION DE AGUASCA. LIENTES, AGUASCALIENTES, UNIVERSIDAD AUTÓNOMA DE AGUASCALIENTES/OBISPADO DE AGUASCALIENTES/ UNIVERSIDAD DE GUADALAJARA, 1999, 456 PP.

JESÚS ANTONIO DE LA TORRE RANGEL

\section{9}

esús Antonio de la Torre Rangel

EL DERECHO A LA EDUCACION EN AGUASCALIENTES. AGUASCALIENTES, UNIVERSIDAD AUTONOMA DE AGUASCALIENTES/INSTITUTO DE EDUCACIÓN DE AGUASCALIENTES, 1999. 100 PP.

BONIFACIO BARBA

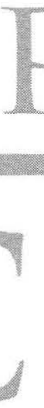

251

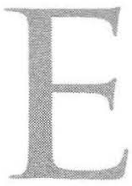

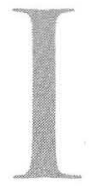

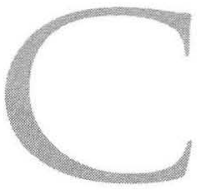




\section{CALEIDOSCOPIO}

REVISTA SEMESTRAL DE CIENCIAS SOCIALES Y H U MAN IDADES

PUBLICADA POR LA UNIVERSIDAD AUTÓNOMA DE AGUASCALIENTES

111111111111111111111

DIRECTOR Jesús Gómez Serrano

SECRETARIO DE REDACCIÓN Enrique Luján Salazar

CONSEJO EDITORIAL

Dr. Bonifacio Barba Casillas (UAA)

Dr. Mauricio Beuchot Puente (IFF-UNAM)

Mtro. Jesús Antonio de la Torre Rangel(UAA)

Dr. Enrique Florescano Mayet (Coordinación Nacional de Proyectos Históricos del conaculta)

Dr. Víctor M. González Esparza (UAA)

Dr. Jorge González Sánchez (U de C)

Lic. Felipe Martínez Rizo (UAA)

Dr. Rollin Kent Serna (CINVESTAV del IPN)

Dr. Jean Meyer Barth (CIDE)

Dra. Yolanda Padilla Rangel (UAA)

Dr. Fernando Pozos (U de G)

Mtra. Guadalupe Ruiz Cuéllar (UAA) Mtra. Sylvia Schmelkes del Valle (CINVESTAV del IPN)

Arq. Marco Alejandro Sifuentes (UAA)

Dr. Genaro Zalpa Ramírez (UAA)

Dirigir correspondencia y colaboraciones a: CALEIDOSCOPIO

Universidad Autónoma dE AGUASCALIENTES,

Edificio 105, Av. Universidad 940, 20100 Aguascalientes, Ags.

DISEÑO GRÁFICO ORIGINAL DE Natalia Rojas Nieto

SUSCRIPCION ANUAL: en México: 60 pesos en el extranjero: 20 USD NÚMERO SUELTO: 30 pesos

ISSN 1405-7107

Certificado de Reserva de Derechos al uso exclusivo, núm. 000487/98

Certificados de Licitud de Título número 10619 y de Licitud de Contenido número 8597 , otorgados por la Comisión Calificadora de Publicaciones y Revistas Ilustradas de la Secretaría de Gobernación 